tionsmaximum $^{[7]}$ von 5 ist gegenüber dem von 1 bathochrom, die Frequenz der $\mathrm{C}=\mathrm{C}$-Schwingung zu geringeren Wellenzahlen verschoben - in Einklang mit den Befunden an 2-4.

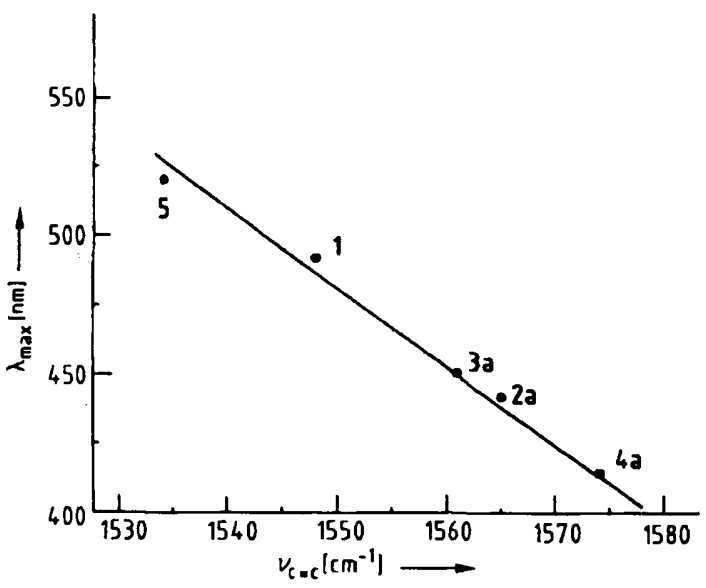

Abb. 1. Beziehung zwischen den $\mathrm{C}=\mathrm{C}$-Streckschwingungsfrequenzen und den Absorptionsmaxima bei den Modellverbindungen 1, 2a, 3a, 4a und 5 .

Die $\mathrm{C}=\mathrm{N}^{\oplus}$-Streckschwingungsfrequenz wird durch Protonierung der Dimethylaminogruppen in Nachbarschaft zum Polyengerüst in 2-5 nicht signifikant geăndert (Tabelle 1). Dies ist in Einklang mit dem Befund, daß in Sehpigmenten $v_{\mathrm{C}-\mathrm{N}}$ unabhăngig von $\lambda_{\max }$ ist ${ }^{[8]}$.

Nach unseren Ergebnissen verringert eine positive Ladung in direkter Nachbarschaft zum Retinalpolyen die Ladungsdelokalisierung, was die $\mathrm{C}=\mathrm{C}$-Schwingungsfrequenz erhöht. Eine externe positive Ladung nahe dem Alkylidenammonium-Ion (5) erhőht die Ladungsdelokalisierung und die Bindungsalternanz, was zu einer Frequenzerniedrigung der $\mathrm{C}=\mathrm{C}$-Streckschwingung führt. Eine externe positive Ladung beeinflußt also nicht nur den angeregten Zustand, sondern auch den Grundzustand.

Nach dem für Bacteriorhodopsin entwickelten Modell der externen Punktladung befindet sich eine negative Ladung nahe dem Cyclohexenteil des Retinalgerüstes, so daß eine Wechselwirkung zwischen Punktladung und Chromophor stattfinden kann. Die von uns untersuchten Modellverbindungen sollten wegen der externen positiven Ladung gegensătzliche Effekte zeigen. Unsere Befunde sind in Einklang mit dem Modell der externen Punktladung und stützen die Annahme, da $B$ die Unterschiede in den $\mathrm{C}=\mathrm{C}$ Streckschwingungsfrequenzen von Bacteriorhodopsin und Sehpigmenten einschließlich der photochemischen Zwischenprodukte durch „through space"-Wechselwirkungen von Chromophor und externen negativen Ladungen zustande kommen.

Eingegangen am 4. Mai, in veranderter Fassung am 28. Juni 1984 [Z 820]

[1] A. Lewis, J. Spoonhower, R. A. Bogomolni, R. H. Lozier, W. Stoeckenius, Proc. Natl. Acad. Sci. USA 71 (1974) 4462; B. Aton, A. G. Doukas, R. H. Callender, B. Becher, T. G. Ebrey, Biochemistry 16 (1977) 2995; K. J. Rothschild, H. Marrero, Proc. Natl. Acad. Sci. USA 79 (1982) 4045; K. Bagley, G. Dollinger, L. Eisenstein, A. K. Singh, L. Zimanzi, ibid. 79 (1982) 4972.

(2) M. A. Marcus, A. Lewis, Biochemistry 17 (1978) 4722; R. Callender, B. Honig, Annu. Rev. Biophys. Bioeng. 6 (1977) 33.

[3] L. Rimai, D. Gill, J. L. Parsons, J. Am. Chem. Soc. 93 (1971) 1353; L. Rimai, M. E. Heyde, D. Gill, ibid. 95 (1973) 4493; M. Heyde, D. Gill, R. Kilponen, L. Rimai, ibid. 93 (1971) 6776.
[4] B. Honig, U. Dinur, K. Nakanishi, V. Balogh-Nair, M. A. Gawinovicz, M. G. Motto, J. Am. Chem. Soc. 101 (1979) 7084; K. Nakanishi, V. BaloghNair, M. Arnaboldi, K. Tsujimoto, B. Honig, ibid. 102 (1980) 7915.

[5] M. Sheves, T. Baasov, Tetrahedron Lett. 24 (1983) 1745.

[6] M. Sheves, T. Baasov, N. Friedman, J. Chem. Soc. Chem. Commun. 1983. 77.

[7] 1. Tabushi, Y. Kuroda, K. Shimokoawa, J. Am. Chem. Soc. 101 (1979) 4759; I. Tabushi, K. Shimokawa, ibid. 102 (1980) 5400.

[8] P. E. Blatz, J. H. Mohler, Biochemistry 14 (1975) 2304: H. Kakitani, T. Kakitani, H. Rodman, B. Honig, R. Callender, J. Phys. Chem. 87 (1983) 3620.

\section{Stabilisierung von Schwefelmonoxid durch Koordination an Ubergangsmetalle**}

\section{Von Wolfdieter A. Schenk*, Johanna Leißner und Christian Burschka}

Schwefelmonoxid, SO, ist eines der klassischen, instabilen kleinen Moleküle ${ }^{[1,2]}$; wir berichten hier uber seine Stabilisierung durch Einbau in Rhodium- und Iridiumkomplexe. Zwar sind Verbindungen mit einer SO-Brücke zwischen $\mathrm{zwei}^{(3)}$ und drei ${ }^{[4]}$ Metallzentren bekannt, doch sind sie, in Einklang mit ihrer Bildungsweise, eher als oxidierte Sulfidokomplexe anzusehen; die Konstitution zweier Komplexe mit terminalen SO-Liganden ${ }^{[5,6]}$ ist noch nicht zweifelsfrei geklärt. Komplexstabilisiertes $\mathrm{S}_{2} \mathrm{O}$ und $\mathrm{S}_{2} \mathrm{O}_{2}$ erhălt man durch Oxidation von $\left[\left(\text { diphos) }{ }_{2} \mathrm{IrS}_{2}\right]^{\oplus}\right.$ (diphos $\left.=\mathrm{Ph}_{2} \mathrm{PCH}_{2} \mathrm{CH}_{2} \mathrm{PPh}_{2}\right)^{16,7]}$.

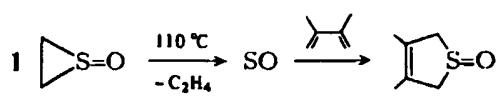

Schwefelmonoxid kann in Lösung durch Thermolyse von Thiiran-S-oxid 1 erzeugt $^{[8]}$ und z. B. mit 1,3-Dienen abgefangen werden ${ }^{[9]}$. Wir haben SO-Komplexe durch Fragmentierung von 1 in der Koordinationssphäre eines Übergangsmetalls hergestellt; SO wird bei seiner Entstehung stabilisiert ${ }^{[5]}$. Setzt man die in Lösung leicht erhăltlichen 14-Elektronen-Komplexe 2 und $3^{[10]}$ bei $-70 \mathrm{bzw}$. $0^{\circ} \mathrm{C}$ mit $1 \mathrm{um}$, so erhălt man die tiefgrünen kristallinen Produkte 4 bzw. 5 (korrekte Elementaranalyse) ${ }^{[1]}$. ${ }^{31} \mathrm{P}$. NMR-spektroskopisch konnten wir bei tiefer Temperatur instabile Zwischenprodukte nachweisen, bei denen es sich vermutlich um Addukte von 1 an die koordinativ ungesăttigten Komplexe 2 und 3 handelt.

$$
\begin{aligned}
& {\left[\mathrm{M}\left(\mathrm{PiPr}_{3}\right)_{2} \mathrm{Cl}\right] \stackrel{+1}{\longrightarrow}\left\{\left[\mathrm{M}\left(\mathrm{PiPr}_{3}\right)_{2}\left(\mathrm{C}_{2} \mathrm{H}_{4} \mathrm{SO}\right) \mathrm{Cl}\right]\right.} \\
& \begin{array}{l}
\text { 2, M-Rh;3, M=Ir } \\
\stackrel{-\mathrm{C}_{2} \mathrm{H}_{4}}{\longrightarrow}\left[\mathrm{M}\left(\mathrm{PiPr}_{3}\right)_{2}(\mathrm{SO}) \mathrm{Cl}\right] \\
4, \mathrm{M}=\mathrm{Rh} ; 5, \mathrm{M}=\mathrm{Ir}
\end{array}
\end{aligned}
$$

Nach spektroskopischen Untersuchungen (Tabelle 1) haben die neuen Verbindungen eine quadratisch-planare Struktur mit trans-Anordnung der sperrigen Phosphanliganden und gewinkelter $\mathbf{M}-\mathbf{S}=0$-Einheit. Bestătigt wird diese Vorstellung durch die Röntgen-Strukturanalyse des Iridium-Komplexes 5 (Abb. 1). Das Metallatom befindet sich in exakt planarer, nahezu quadratischer Umgebung [Abstand Ir-P 237.1(2), Ir-Cl und Ir-S 223.6(3) pm (Fehlordnung zwischen $\mathrm{Cl}$ und $\mathrm{S}$ in der Raumgruppe $\mathrm{P} 2,1 \mathrm{c})$ ].

[*] Prof. Dr. W. A. Schenk, Dipl.-Chem. J. LeiBner, Dr. C. Burschka Institut für Anorganische Chemie der Universitat Am Hubland, D-8700 Warzburg

[**] Diese Arbeit wurde von den Firmen Ciba-Geigy AG und Degussa AG sowie vom Fonds der Chemischen Industrie unterstutzt. 


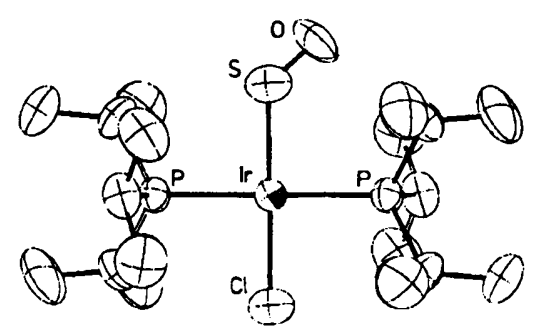

Abb. 1. Struktur des Komplexes 5 im Kristall. Raumgruppe $P_{2} / c$ (Nr. 14), $Z=2, a=8.128(3), b=9.075(3), c=16.659(4) \hat{A}, \beta=93.18(3)^{\circ}, \rho_{\text {her }}=1.62$, $\rho_{\text {axp }}=1.615 \mathrm{mg} / \mathrm{mm}^{3}, R_{1}=0.028(0.034), R_{2}=0.036(0.037)$ for 1772 (2167) Reflexe $\left(I_{o b} \geq 3 \sigma(D)\right)$. Weitere Einzelheiten zur Kristallstrukturuntersuchung konnen beim Fachinformationszentrum Energie Physik Mathematik, D-7514 Eggenstein-Leopoldshafen 2, unter Angabe der Hinterlegungsnummer CSD 51005 , der Autoren und des Zeitschriftenzitats angefordert werden.

Die SO-Gruppe [Abstand S-O 132(2) pm, Winkel Ir-S-O $\left.130(1)^{\circ}\right]$ ist um ca. $20^{\circ}$ aus der Koordinationsebene herausgedreht. Damit sind 4 und 5 mit den entsprechenden $\mathrm{SO}_{2}$ Komplexen ${ }^{[12]}$ strukturell eng verwandt.

Tabelle 1. Spektroskopische Daten der SO-Komplexe 4 und 5.

\begin{tabular}{|c|c|c|c|c|}
\hline $\begin{array}{l}\text { Kom- } \\
\text { plex }\end{array}$ & $\begin{array}{l}\text { IR (Nujol) } \\
{\left[\mathrm{cm}^{-1}\right]}\end{array}$ & $\delta$ & 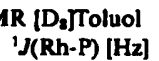 & $\begin{array}{l}\mathrm{MS} \\
m / z\left(M^{\bullet}\right)\end{array}$ \\
\hline 4 & $\begin{array}{l}1075 \text { \& } \vee(\mathrm{SO}) \\
455 \mathrm{~m} \delta(\mathrm{RhSO}) \\
341 \mathrm{~m} \text { (RhCl), } \\
271 \mathrm{~m} \text { (RhS) }\end{array}$ & 42.4 & 153 & 506 \\
\hline 5 & $\begin{array}{l}1071 \text { s } v(S O) \\
475 \mathrm{~m} \delta \text { (IrSO), } \\
337 \mathrm{~m} \text { (IrCl), } \\
279 \mathrm{~m} \text { (IrS) }\end{array}$ & 28.1 & & 596 \\
\hline
\end{tabular}

Eingegangen am 24. Mai, ergänzt am 31. Juli 1984 [Z 849]

[1] P. W. Schenk, Z. Anorg. Allg. Chem. 211 (1933) 150; P. W. Schenk, R. Steudel, Angew. Chem. 77 (1965) 437; Angew. Chem. Int. Ed. Engl. 4 (1965) 402.

[2] Gmelin Handbuch der Anorganischen Chemie, 8. Aufl., Schwefel, Ergănzungsband 3 (Schwefeloxide), Springer, Bertin 1980, S. $40 \mathrm{fr}$.

(3) M. Honler, A. Baitz, Chem. Ber. 109 (1976) 3147.

[4] L. Mark6, B. Marko-Monostory, T. Madach, H. Vahrenkamp, Angew. Chem. 92 (1980) 225; Angew. Chem. Int. Ed. Engl. 19 (1980) 226; A. Winter, L. Zsolnai, G. Huttner, J. Organomet. Chem. 234 (1982) 337; I. P. Lorenz, J. Messelhăuser, Z. Naturforsch. B 39 (1984) 403.

[5] Agarwala et al. erzeugten SO aus Epithiostilben-S-oxid bei $30^{\circ} \mathrm{C}$ in $\mathrm{Ge}-$ genwart von $\left[\mathrm{Rh}\left(\mathrm{PPh}_{3}\right)_{3} \mathrm{X}\right](\mathrm{X}-\mathrm{Cl}, \mathrm{Br})$ und isolierten $\mathrm{Komplexe}$, die sie nach Elementaranalyse und IR-Spektrum als $\left[R h\left(P P h_{3}\right)(S O)(\mu-X)\right)_{2}$ beschrieben: K. S. Arulsamy, K. K. Pandey, U. C. Agarwala, Inorg. Chim. Acta SA (1981) LS1.

16] G. Schmid, G. Ritter, T. Debaendemaeker, Chem. Ber. 108 (1975) 3008.

(7) G. Schmid, G. Ritter, Angew. Chem. 87 (1975) 673; Angew. Chem. Int. Ed. Engl. 14 (1975) 645.

[8] G. E. Hartzell, J. N. Paige, J. Am. Chem. Sac. 88 (1966) 2616.

(9) R. M. Dodson, R. F. Sauers, Chem. Commun. 1967, 1189.

[10] C. Busetto, A. D'Alfonso, F. Maspero, G. Perego, A. Zazzetta, J. Chem. Soc. Dalion Trans. 1977, 1828; H. L. M. van Gaal, J. P. J. Verlaan, J. Organomet. Chem. 133 (1977) 93; H. L. M. van Gaal, F. L. A. van den Bekerom, ibid. 134 (1977) 237; R. Meij, D. J. Stufkens, K. Vrieze, W. van Gerresheim, C. H. Stam, ibid. 164 (1979) 353.

[11] $250 \mathrm{mg}(0.5 \mathrm{mmol})\left(\left(\mathrm{C}_{\mathrm{H}} \mathrm{H}_{14}\right)_{2} \mathrm{RhCl}_{2}\right.$ werden in $15 \mathrm{~mL}$ Toluol mit $0.4 \mathrm{~mL}$ (2.0 mmol) PiPr, nach [10] zu 2 umgesetzt. Man kublt auf $-70^{\circ} \mathrm{C}$, setzt $0.06 \mathrm{~mL}$ (1.0 mmol) $1 \mathrm{zu}$ und laBt bei dieser Temperatur stehen. Nach drei Tagen scheidet sich 4 in Form tiefgroner Kristalle ab, die mit Hexan gewaschen und im Vakuum getrocknet werden. Ausbeute $300 \mathrm{mg}$ (59\%), Fp $=110^{\circ} \mathrm{C}$ (Zers.). Analog erhalt man den Ir-Komplex 5 bei $0^{\circ} \mathrm{C}$ in $68 \%$ Ausbeute als grinschwarze Kristalle, $\mathrm{Fp}=155^{\circ} \mathrm{C}$ (Zers.).

[12] O. J. Kubss, R. R. Ryan, Inorg. Chim. Acta 47 (1981) 131.
Leichte Rotation um die exocyclische

Kohlenstoff-Kohlenstoffbindung in

Lithium-a-aminoenolaten: ein Vergleich **

Von Gernot Boche*, Ferdinand Bosold und Robert Eiben

In den $\alpha$-Aminoenolaten („Amidenolaten“) 1, 2 und 3 fanden wir erstmals eine leichte Rotation um die exocyclische CC-,Doppelbindung "[1] dieser Verbindungsklasse.<smiles>CO[As](N)c1ccccc1</smiles>

1<smiles>COc1ccccc1I</smiles>

2

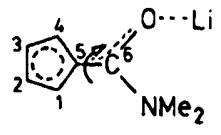

3
Die $100 \mathrm{MHz}-{ }^{1} \mathrm{H}-\mathrm{NMR}-S p e k t r e n$ von Lithium-dimethylamino(cycloheptatrienyliden)methanolat 1 sind temperaturabhăngig (Abb. 1) ${ }^{[2]}$. Bei $39^{\circ} \mathrm{C}$ sind die Signale von $\mathrm{H}^{\prime}$ und $\mathrm{H}^{6}(\delta=5.17$ und 4.93; eine Zuordnung wurde nicht vorgenommen) getrennt, während sie aufgrund der schnellen Rotation um die $\mathrm{C}^{7} \mathrm{C}^{8}$-Bindung bei $70^{\circ} \mathrm{C}$ koaleszieren und bei $83^{\circ} \mathrm{C}$ zu einem Dublett aufspalten. Fur die Rotationsbarriere in 1 errechnet sich daraus die freie Aktivierungsenthalpie $\mathrm{zu} \Delta G^{+}\left(70^{\circ} \mathrm{C}\right)=17.5 \pm 0.5 \mathrm{kcal} / \mathrm{mol}$. Ähnlich wurde die Barriere für die Rotation um $\mathrm{C}^{7} \mathrm{C}^{8}$ im dihydroanalogen 2 ermittelt $\left(\Delta G^{+}\left(25^{\circ} \mathrm{C}\right)=14.0 \pm 0.5\right.$ $\mathrm{kcal} / \mathrm{mol}$ ). Die Rotation um $\mathrm{C}^{5} \mathrm{C}^{6}$ in 3 konnte dagegen selbst bei $-106^{\circ} \mathrm{C}$ nicht eingefroren werden, d.h. $\Delta G^{+}\left(-106^{\circ} \mathrm{C}\right)<8 \mathrm{kcal} / \mathrm{mol}^{[3,4]}$.

In 2 ist die negative Ladung im gekreuzt konjugierten Pentadienid-Teil stabilisiert, in 3 im Cyclopentadienid-

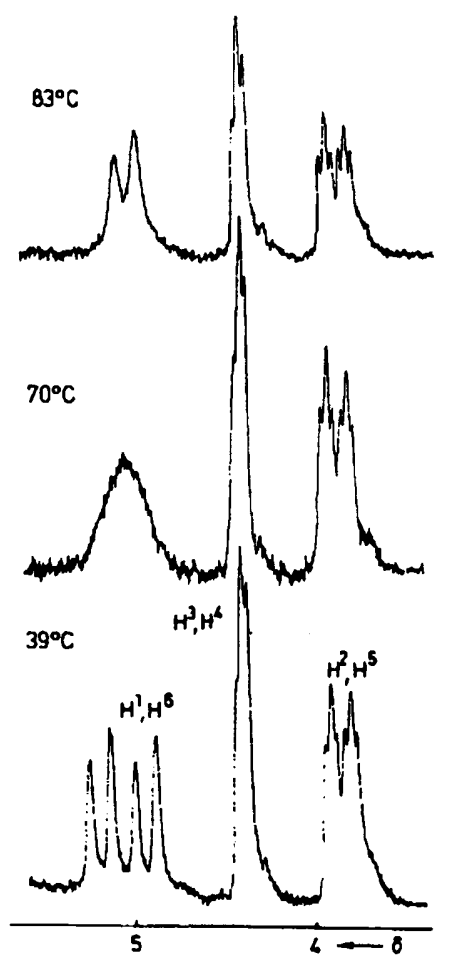

Abb. 1. ${ }^{1} \mathrm{H}-\mathrm{NMR}-\mathrm{Spektren}\left(100 \mathrm{MHz}\right.$ ) von 1 in $\left[\mathrm{D}_{2}\right]$ Tetrahydrofuran (THF) bei 83,70 und $39^{\circ} \mathrm{C}$.

[*] Prof. Dr. O. Boche, F. Bosold, Dipl.-Chem. R. Eiben

Fachbereich Chemie der Universit

Hans-Meerwein-StraBe, D-3550 Marburg

[**] Diese Arbeit wurde vom Fonds der Chemischen Industrie unterstutzt. 